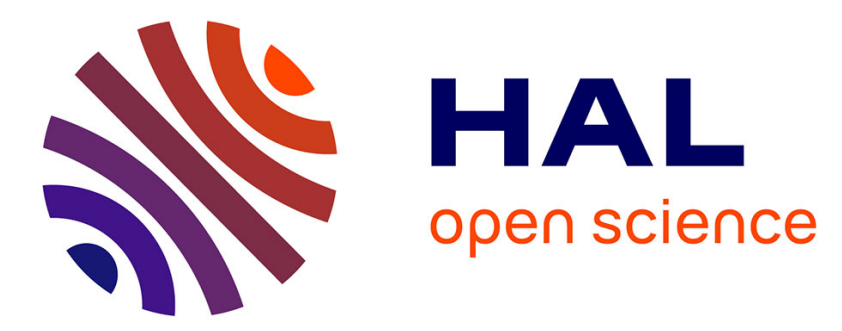

\title{
Le sens des lieux. Espaces et pratiques dans les palmeraies du Jérid (Sud-ouest tunisien)
}

\author{
Vincent Battesti, Nicolas Puig
}

\section{To cite this version:}

Vincent Battesti, Nicolas Puig. Le sens des lieux. Espaces et pratiques dans les palmeraies du Jérid (Sud-ouest tunisien). Journal d'Agriculture Traditionnelle et de Botanique Appliquée, 1999, 41 (2), pp.19-44. 10.3406/jatba.1999.3710 . halshs-00004075v4

\section{HAL Id: halshs-00004075 \\ https://shs.hal.science/halshs-00004075v4}

Submitted on 12 Jan 2016

HAL is a multi-disciplinary open access archive for the deposit and dissemination of scientific research documents, whether they are published or not. The documents may come from teaching and research institutions in France or abroad, or from public or private research centers.
L'archive ouverte pluridisciplinaire HAL, est destinée au dépôt et à la diffusion de documents scientifiques de niveau recherche, publiés ou non, émanant des établissements d'enseignement et de recherche français ou étrangers, des laboratoires publics ou privés.

\section{(1) (1) $\$$}

Distributed under a Creative Commons Attribution - NonCommercial - NoDerivatives 44.0 


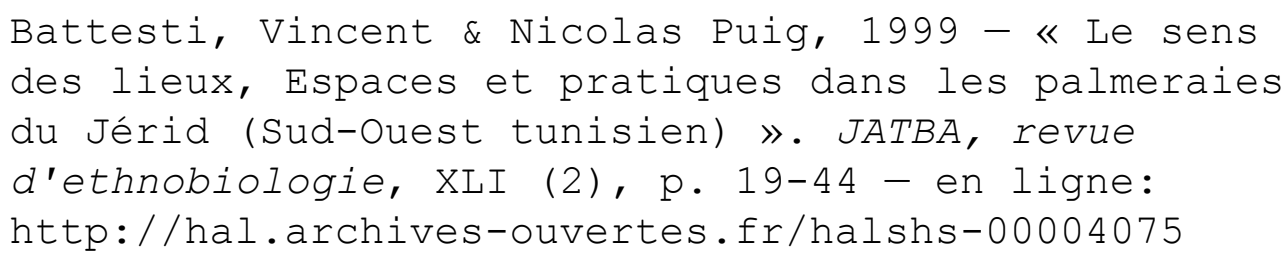

\section{LE SENS DES LIEUX}

\section{ESPACES ET PRATIQUES DANS LES PALMERAIES DU JÉRID (SUD-OUEST TUNISIEN)}

\section{Vincent Battesti” et Nicolas Puig**}

RÉSUMÉ.- Dans les oasis du Jérid (Sud-Ouest tunisien), l'emprise humaine se révèle à travers diverses pratiques et représentations symboliques. Les spatialités et les temporalités se combinent pour créer les échelles de la palmeraie. L'intensité de la présence humaine est tangible dans la minutie des activités agricoles; elle transparaît également à travers les sociabilités qu'abritent les jardins, les manières de nommer les lieux, ou encore les diverses modalités d'expression esthétique qui se font jour, même au sein du travail.

MOTS-CLEFS.- Lieux - spatialité - temporalité - espace - oasis - palmeraie - Jérid - Tunisie.

ABSTRACT.- In the oases of Jerid (S.W. of Tunisia), the human sway is revealed through various practices and symbolic representations. Spatial and temporal activities are combined to create scales of the palm-grove. The intensity of the human presence is visible in the meticulousness of the agricultural activities; this is displayed also through the "sociabilities" practised in the gardens, the way to give name to places, or the modes of aesthetic expressions, even in the work.

KEY-WORDS.- Spatiality - Temporality - Space - Oasis - Palm-grove - Jerid - Tunisia.

La palmeraie, « nature domestique " adossée au bâti, mérite un regard qui ne se restreint pas à la seule agriculture. Grâce à la richesse et la diversité des activités qu'elle suscite, cette «forêt » du monde aride peut également faire l'objet d'une approche spécifique, s'émancipant, le temps de l'analyse, de l'ensemble oasis (wâh'a) qui les englobe, elle et la ville, dans une totalité articulée. Cet espace dont la complexité sociale et écologique est si souvent soulignée ${ }^{2}$ ne manque pas de susciter dans nos imaginaires (occidentaux) de multiples visions plus ou moins erronées y compris au sujet de ses performances agronomiques. Aussi, la «charge »

\footnotetext{
* Anthropologue, Musean (MNHN).

** Anthropologue, CEDEJ du Cairc.

'Selon l'expression de Philippe Descola (1986).

2 Par exemple dernièrement Kassah, $1996: 12$.
} 
symbolique attachée à l'oasis incite, là plus qu'ailleurs, à la prudence et à la circonspection $^{3}$.

S'agissant de ces lieux si fortement façonnés par une histoire qui se perd dans l'imaginaire mythique, l'étude anthropologique des pratiques extra-agricoles n'a pas encore été entreprise de façon systématique. Pourtant, dans leur fréquentation de la palmeraie, les hommes font plus que mettre en œuvre une unique logique productive.

Diverses représentations symboliques émergent de l'emprise humaine, qui associent spatialités et temporalités aux différentes échelles de la palmeraie. L'intensité de la présence humaine est certainement discernable dans la minutie des activités agricoles. Elle transparaît également à travers les sociabilités qu'abritent les jardins, les manières de nommer les lieux ${ }^{4}$, ou encore les diverses modalités d'expression esthétique qui se font jour, parfois au sein même du travail.

C'est par l'analyse du rapport à l'espace et au temps ${ }^{5}$ que nous entreprenons cette visite des palmeraies, afin de rendre compte de l'enchevêtrement dans un même lieu de diverses dimensions de l'existence collective.

En privilégiant cette approche, nous espérons dévoiler une partie des relations que les hommes entretiennent avec les lieux qu'ils produisent. En somme, nous invitons à un basculement du lieu anthropologique - «le lieu commun à l'ethnologue et à ceux dont il parle (...) celui qu'occupent les indigènes qui $y$ vivent » (Augé, 1992: 57) - à l'anthropologie du lieu dans laquelle l'espace devient le point central des investigations.

Le Jérid, région située au sud-ouest de la Tunisie (document 1), est une terre célèbre de phoniciculture. Les plus anciennes de ses palmeraies (Nefta, Tozeur, al-Hamma, al-Oudiane) datent probablement de l'Antiquité ${ }^{6}$. Aux côtés de ces palmeraies, des créations plus récentes ont vu le jour sous le signe de la rationalité agronomique, à l'instigation des autorités coloniales et de quelques agriculteurs étrangers, puis sous l'impulsion du jeune État tunisien.

${ }^{3}$ Ainsi, J. Bisson (1995), citant des études de C. Riou et de D. Dubost, relativise l'idée de la performance agronomique de la palmeraie.

${ }^{4}$ Parlant des lieux, Jean-Charles Depaule (1991:9) note que « le langage quotidien ne cesse en les nommant et en les surnommant, en les qualifiant, de les distinguer, de les identifier et de les mettre en relation $"$.

${ }^{5}$ G. Condominas $(1980: 20)$ fait des relations à l'espace et au temps « écologiques » la base de l'espace social d'une société, déplorant que trop souvent "l'examen du milieu habité et exploité par un groupe » soit " détaché de celui du temps... ».

${ }^{6}$ Voir par exemple Paty de Clam, 1890. 


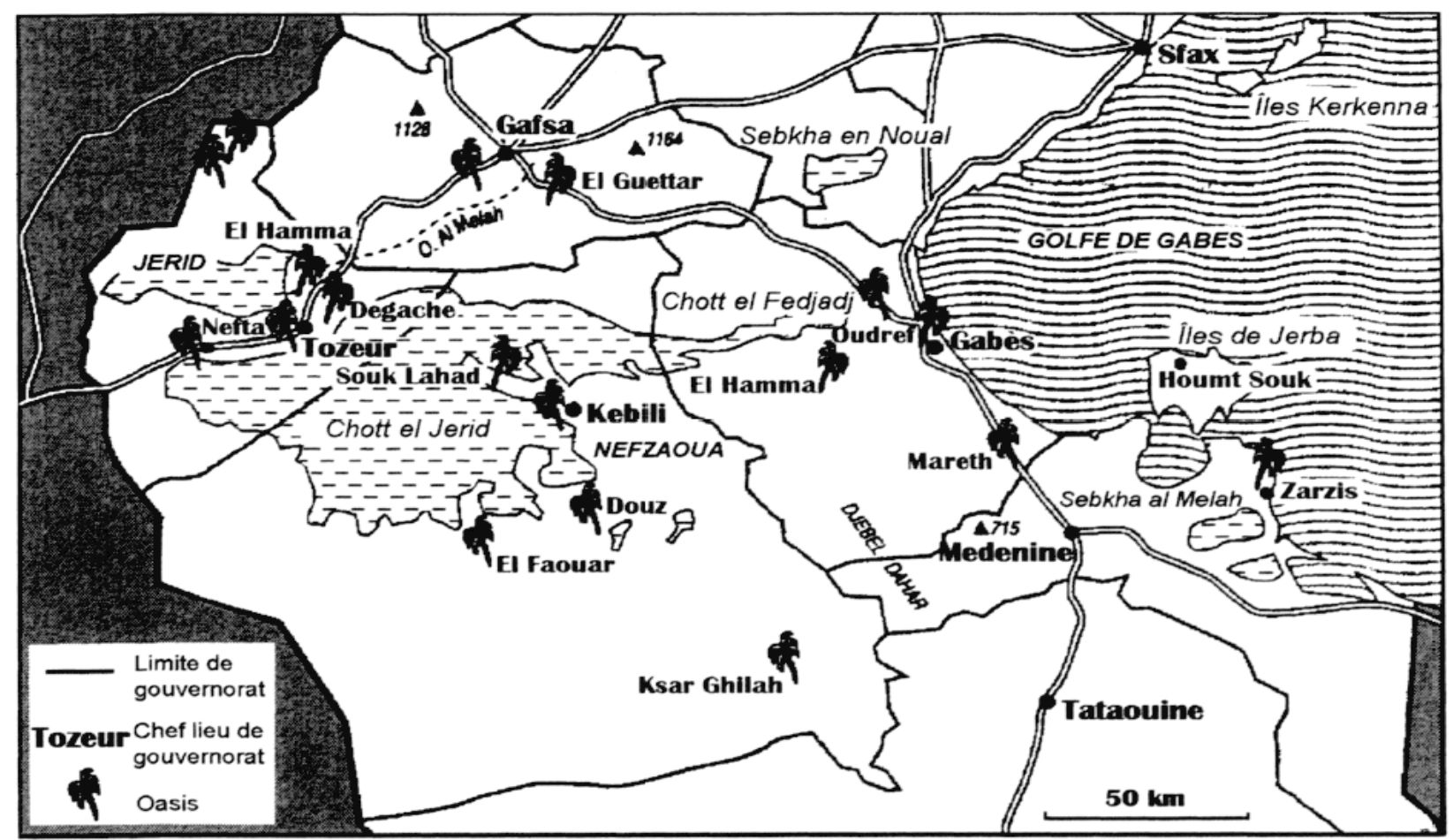

Document 1 : Le Sud tunisien (Source : Puig, 1997, modifié)

C'est vers les vieilles palmeraies enracinées autour de leurs cités que nous porterons principalement notre regard sans négliger les créations récentes, autour desquelles, bien souvent, se sont fixés des nomades accédant à la propriété. Ces nouvelles palmeraies représentent aujourd'hui la majeure partie des périmètres irrigués ${ }^{7}$.

\section{L'ORGANISATION SPATIALE DE LA PALMERAIE}

Le plan des palmeraies évolue, il s'agit d'espaces mouvants produits par la rencontre de facteurs socio-historiques et de contingences écologiques. La fonction de production agricole de la palmeraie est bien sûr importante, cependant d'autres activités sont venues au cours de l'histoire enrichir les productions des oasis du Jérid, comme le commerce et de manière plus générale les échanges divers permis par leur situation géographique ${ }^{8}$. Les pratiques religieuses s'y sont développées, au sein même des palmeraies qui abritent une multiplicité d'espaces sacrés.

${ }^{7}$ Les anciennes oasis couvrent 3300 ha pour un total de 7819 ha irrigués au Jérid. Les périmètres restant sont constitués de palmeraies de création récente. Source : CRDA Tozeur, 1996.

8 « Le Grîd est décrit par les chroniqueurs et voyageurs arabes et occidentaux depuis le "Moyen Âge" jusqu'à l'époque moderne comme étant une région particulièrement riche dont les activités économiques sont à la fois variées et lucratives » (Henia, 1980). 
La logique de l'organisation spatiale dans les palmeraies est celle de l'emboîtement : l'espace se structure à partir d'unités de plus en plus englobantes, depuis la planche de culture (ou d'irrigation) jusqu'au jar, le terroir. Les grandes divisions de la palmeraie suivent l'axe de l'oued dont les ramifications délimitent les différents quartiers d'oasis.

\section{Le jar, ses subdivisions et ses extensions}

\section{STRUCTURE SPATIALE DU TERROIR}

Le terme jar désigne la palmeraie ancienne et un terroir local bien délimité. Aussi, n'est-il pas usité dans les nouvelles palmeraies où seul le toponyme est employé. La circulation de l'eau constitue une des structurations de la palmeraie dans laquelle elle dessine un système organique. Face au réseau des séguias organisées en arborescence s'imbrique celui de drains (khandig, plur. khanâdig), car il est capital que l'eau soit correctement amenée puis évacuée sans stagner dans les parcelles.

Les voies et les chemins de terre constituent aussi les artères du jar. Leur tracé a pu évoluer au gré de la redéfinition des formes des propriétés dans la palmeraie, mais également avec l'introduction des charrettes, semble-t-il à la fin du protectorat $^{10}$.

Le jar est divisé en grandes parties (ou quartiers) désignées par le même terme de « jar » suivi du toponyme. La palmeraie de Tozeur est ainsi divisée en trois jar: jar ${ }^{c}$ Abbas, jar al-Wasat, jar ar-Ribat auxquels il faut ajouter les expansions plus récentes comme Helba et Jehim (document 2).

Les extensions (mazyûd) effectuées par les particuliers proposent une autre logique de l'organisation spatiale des cultures ${ }^{11}$. Ce sont des jardins plantés dans leur immense majorité en palmiers dattiers deglet nour ${ }^{12}$ disposés en lignes à intervalles de 6 à 10 mètres. Toujours en bordure de palmeraie, les extensions sont soit une propriété qui s'étend sur la terre nue attenante, soit un nouveau jardin. Pour les khammes ${ }^{13}$, l'extension est un moyen peu onéreux d'accéder à la propriété ;

9 Alors que l'on parle du «Jar Tozeur », on dira par exemple, simplement « Nefleyet » où «Ibn Chabbat » qui sont des créations récentes.

10 À Degache, l'élargissement des chemins pour permettre le mouvement des charrettes dans la palmeraie eut lieu en 1965 à la demande des propriétaires. L'oasis d'El Hamma a connu un réaménagement de moindre importance et de nombreuses propriétés ne sont accessibles encore aujourd'hui qu'à pied ou à dos d'âne.

${ }^{11}$ La plupart du temps illégales, elles représenteraient environ 600 ha sur les 7819 ha de palmeraies du Jérid (Source: CRDA Tozeur 1996). Au regard de la région voisine du Nefzaoua, elles constituent un phénomène limité.

12 Cultivar « commercial » : « doigt de lumière » ou « doigt de Nour » (princesse arabe), cette datte a une valeur commerciale importante, car distribuée sur les marchés internationaux.

${ }^{13}$ Le khammesa est une forme de métayage qui couvre de nombreux arrangements différents, la base étant que le khammes (le métayer) exploite une parcelle pour le compte d'un 
certains propriétaires ont recours aux extensions pour rationaliser leur production agricole et déterminer eux-mêmes l'aménagement de leur terre, sans être contraints par le plan pré-existant de la palmeraie.

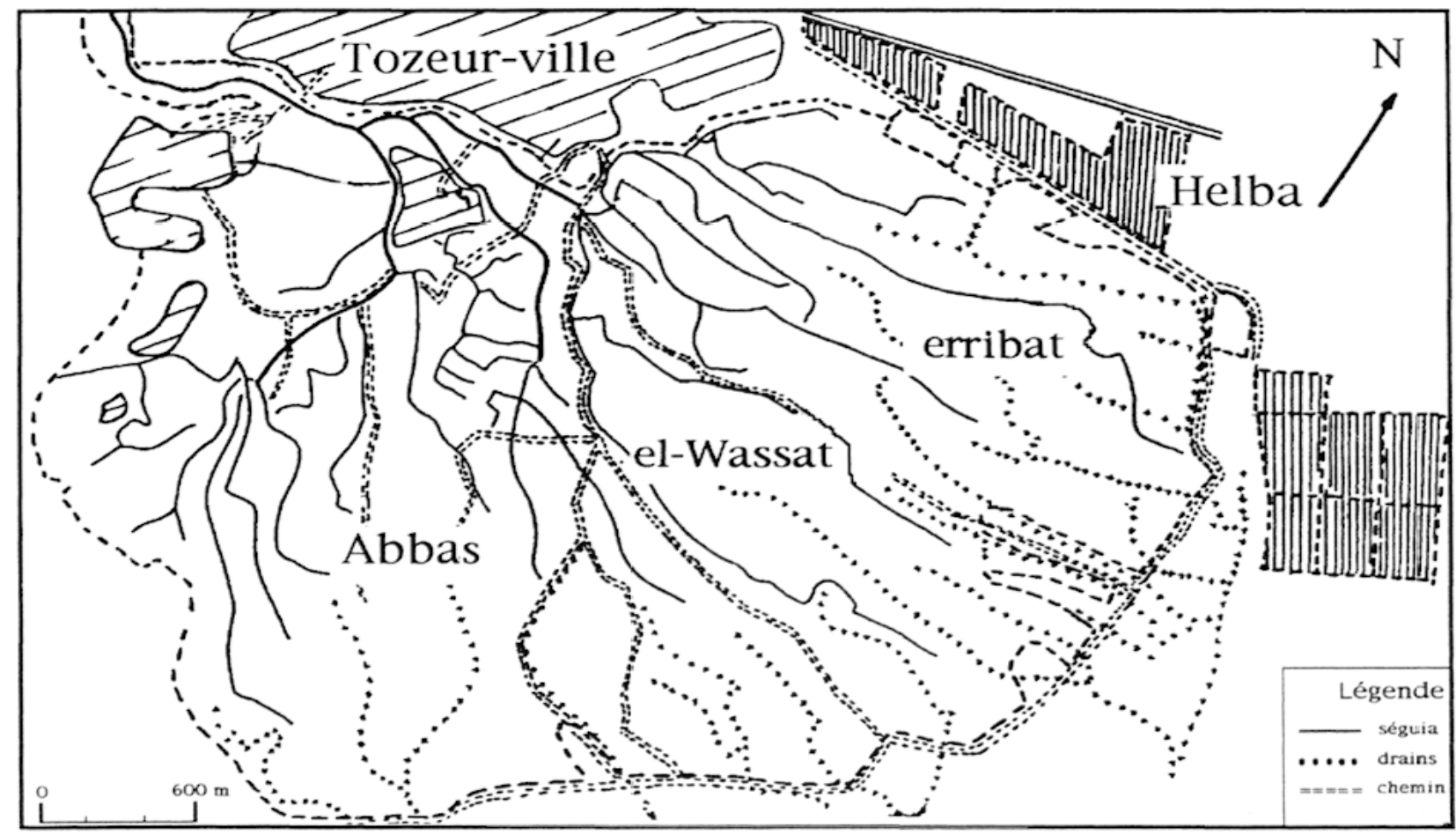

Document 2 : La palmeraie de Tozeur, réseau des séguias et des drains principaux (Source :

A. Kassah, 1991).

L'ordre lignager a longtemps été au principe du fonctionnement des palmeraies : « le langage du lignage s'inscrivait directement dans l'espace cultivé de l'oasis puisqu'il présidait au fonctionnement du système hydraulique " constate Kilani (1992 : 274) à propos de l'oasis d'al-Qsar (région de Gafsa). G. Bédoucha (1987: 282) estime que dans la «presqu'île » du Nefzaoua la réforme du système de distribution de l'eau n'a pas été sans rapport avec la déliquescence du système lignager et de son ordre. Celui-ci, dans l'ensemble des palmeraies, ne s'impose plus comme par le passé depuis que la gestion de l'eau est passée entre les mains de l'État.

Aujourd'hui, il n'est pas possible de trouver trace dans la palmeraie de Tozeur d'un regroupement des jardins appartenant à un même lignage tant l'histoire a travaillé les espaces communautaires ${ }^{14}$. On peut trouver ici et là des regroupements de jardins portant le nom d'une communauté lignagère, comme c'est le cas pour le

propriétaire et reçoive un cinquième de la production de dattes et souvent un « encouragement " pécuniaire mensuel.

14 Il existe bien un jar des Awlâd al-H'aj, mais ses jardins sont disséminés dans toute la palmeraie, le terme jar dans ce cas ne renvoyant pas à un quartier d'oasis. 
jar Beni ${ }^{c}$ Ali de Nefta. Encore faut-il s'interroger sur les réalités auxquelles renvoient aujourd'hui ces appellations, car il y a un « effet d'optique » (de réalité) du nom qui incite à penser que ces espaces (palmeraie comme bâti) forment une homogénéité ethnique ${ }^{15}$ alors qu'ils connaissent désormais une certaine mixité, probablement plus avancée encore dans les jardins ${ }^{16}$.

En revanche, la logique de structuration ethnique de l'espace se retrouve dans certaines créations récentes. La palmeraie de Nefleyet, créée à la fin des années cinquante, est divisée en deux parties. La zone de jardins appartenant aux personnes venant du quartier des Chebbia de Tozeur est appelée shig shebbiya ${ }^{17}$, la partie des Chebbia. L'autre partie ayant été distribuée aux habitants des quartiers centraux est appelée shig al-blad, la partie des habitants du centre-ville.

Des parcelles en périmètres irrigués ont enfin été attribuées par lot à des membres de groupes nomades de la région dans une optique de sédentarisation. Certaines oasis ne sont occupées que par les membres d'un groupe qui s'installe ensemble sur un territoire. Une partie des Awlad Sidi Abid (des Rkerka, principalement) s'est ainsi fixée à Chekmou et une fraction des éleveurs Ghrib à Hezoua.

\section{INDIVIDUALITÉ DES TERROIRS}

Les terroirs du Jérid possèdent des identités très fortement individualisées correspondant à l'émulation - pour ne pas dire à «l'esprit de clocher » ! - qui règne entre les vieilles cités. La variabilité des lexiques de l'agriculture témoigne de ce caractère fortement spécifique de chaque palmeraie, et peut-être d'une histoire différente, sans toutefois renvoyer à des différences techniques majeures.

Le tableau suivant (Tableau 1) regroupe les diverses dénominations de l'espace dans les jardins selon les terroirs. Les planches de culture ou d'irrigation s'imbriquent selon une logique d'emboîtement avec une appellation spécifique pour chaque niveau (cf. document 3 ).

Le niveau d'ordre 1 est la plus petite unité, une cuvette irriguée d'un seul tenant, délimitée d'ados de terre, de forme carrée (a) ou de forme allongée (b). Le niveau d'ordre 2 est l'unité supérieure qui contient celles d'ordre 1 . Il existe parfois un niveau intermédiaire, d'ordre 1 bis, sous-structure occasionnelle de l'ordre 2 ; il s'agit à 1'intérieur du niveau 2 d'un ensemble de structures d'ordre 1 . L'ordre 2 bis est une superstructure occasionnelle du niveau 2 , ensemble de structures de ce niveau.

\footnotetext{
${ }^{15}$ Ethnique désigne dans ce contexte une appartenance fondée sur la communauté lignagère.

${ }^{16} \mathrm{Cf}$. sur ce point Puig, 1997.

17 Il faut noter que dans certains contextes, le terme de shig peut acquérir un sens politique signifiant, ligue, fraction ou partie.
} 


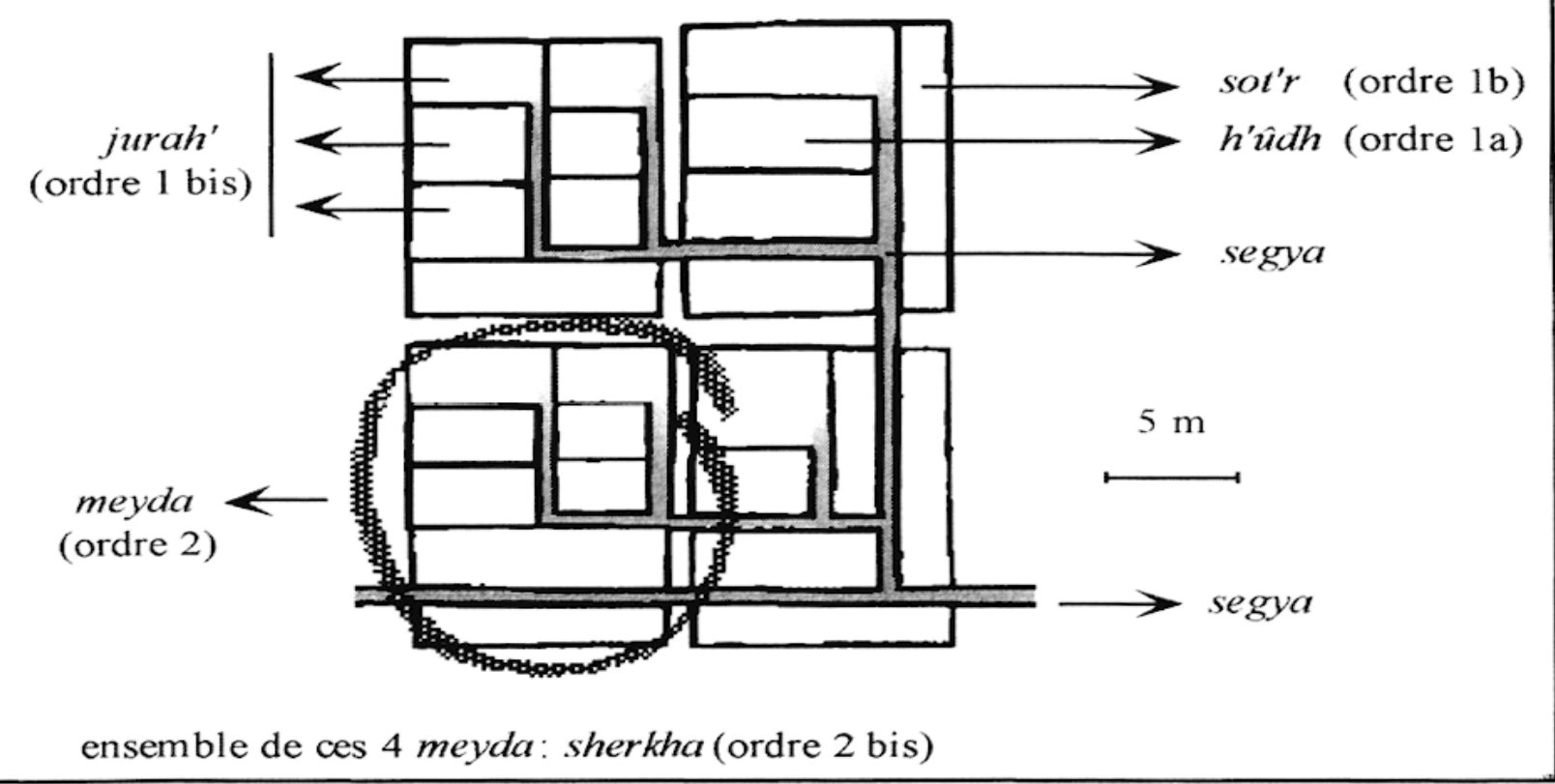

Document 3 : Plan schématique d'une sherkha dans un jardin de Nefta

La terminologie des planches de culture est la plus riche dans les quatre vieilles oasis de la région (les noms de barrages, de séguias et d'ados n'ont pas fait l'objet d'une enquête complète dans les nouvelles palmeraies). Cette richesse lexicale coïncide avec un système général plus complexe que dans les créations récentes. Les termes employés dans les jeunes palmeraies révèlent les liens qu'elles entretiennent avec les formules techniques de jar plus anciens.

Ainsi, Castilia est une palmeraie créée dans les années cinquante. Par sa proximité, elle est dans l'aire d'influence de Tozeur. Nefleyet, datant de la même époque, est travaillée par des agriculteurs de Tozeur et de Degache. La terminologie utilisée par exemple pour désigner le niveau d'ordre 2 est à la fois celle de Tozeur (sherka) et d'al-Hamma (darja); quelquefois, une même personne use des deux.

À Ibn Chabbat, le cas est différent. Il s'agit d'une palmeraie très récente située entre Tozeur et Nefta dont la création remonte à une dizaine d'années. La palmeraie est encore très ouverte (les arbres sont jeunes et la densité est faible) et donc sans une réelle structure oasienne. Seul est en place le niveau d'ordre 1 ( $h$ 'ûdh). On peut émettre l'hypothèse que lors de la fermeture de la palmeraie se produira une complication à la fois des formes et du lexique. 
Tableau 1 : Tableau du lexique des espaces irrigués du jardin et champs sémantiques des termes

\begin{tabular}{|c|c|c|c|c|c|c|c|c|c|c|}
\hline \multirow[b]{2}{*}{$\begin{array}{c}\text { Oasis } \\
\text { I } \\
\text { désignations } \\
\text { planches }\end{array}$} & \multicolumn{4}{|c|}{ Anciennes palmeraies } & \multicolumn{6}{|c|}{ Créations récentes } \\
\hline & Nefta & Tozeur & $\begin{array}{c}\mathrm{Al} \\
\text { Hamma }\end{array}$ & Degache & Castilia & Nefleyet & $\begin{array}{c}\text { Ibn } \\
\text { Chabbat }\end{array}$ & Hazoua & Dghoumes & $\begin{array}{c}\text { Al } \\
\text { Fawar }\end{array}$ \\
\hline Ordre 1 (a) & $h^{\prime} \hat{u} d h$ & meskba & meskba & meskba & meskba & meskba & $h^{\prime} \hat{u} d h$ & $h^{\prime} \hat{u} d h$ & meskba & $h^{\prime} \hat{u} d h$ \\
\hline Ordre 1 (b) & sot'r & $\begin{array}{l}\text { khit / } \\
\text { jurah }\end{array}$ & jurah' & $\begin{array}{l}\text { smat'/ } \\
\text { jabûn }\end{array}$ & khit & & & $\begin{array}{l}\text { smat'/ } \\
\text { sot'r }\end{array}$ & & \\
\hline Ordre 1 bis & jurah' & sherkha & $\begin{array}{l}\text { jurah'/ } \\
\text { jabûn }\end{array}$ & & & & & & & \\
\hline Ordre 2 & meyda & sherkha & darja & darja & & $\begin{array}{l}\text { darja / } \\
\text { sherkha }\end{array}$ & & meyda & darja & $\begin{array}{l}\text { treyda / } \\
\text { h'izân }\end{array}$ \\
\hline Ordre 2 bis & sherkha & & & sherkha & & & & sherkha & & \\
\hline Barrage & $\begin{array}{l}\text { sedd / } \\
\text { miftah' }\end{array}$ & maghleg & & maghleg & & & & & & \\
\hline $\begin{array}{l}\text { Ségya } \\
\text { principal }\end{array}$ & $\begin{array}{l}\text { segyat-al- } \\
\text { hâmala }\end{array}$ & $\begin{array}{l}\text { segyat-al- } \\
\text { um }\end{array}$ & $\begin{array}{l}\text { segyat-al- } \\
\text { um }\end{array}$ & & & & & & & \\
\hline Ados & jiser & mirued & jiser & & & & & & & jiser \\
\hline
\end{tabular}




\section{Quelques notes d'étymologie:}

Darja : marche, palier, degré. R. Dozy (1927) signale le sens de "petit espace de temps », instant. Le terme peut donc renvoyer à une unité de temps nécessaire au remplissage des cuvettes ;

Fum : bouche ;

Hadd : frontière, limite ;

$H^{\prime} \hat{u} d h$ : bassin ;

H'izân: sol dur et inégal ;

Jabûn : long. Terme local dont il n'est fait mention dans aucun dictionnaire ;

Jiser : pont, digue ;

Jurah': blessure, plaie. C'est ce sens qui est retenu dans les jardins récents pour une cuvette hors norme, située en bordure de jardin. On comprend moins le lien entre le sens classique et l'emploi vernaculaire désignant un ensemble de cuvettes en vieilles palmeraies ;

Khit : fil, de coudre ;

Meyda ou mîda: table, au Maghreb, petite table ronde. Dans une acception technique, meyda ma'iya signifie nappe phréatique. Utilisé à Nefta et à Hazoua. Il y a peut-être eu emprunt au langage technique des forages qui se sont multipliés dans la région depuis près d'un siècle ;

Meskba : lieu où l'on verse, de sakaba, verser, irriguer ;

Miftah': clef;

Sedd : barrage ;

Segya al-Hâmala : séguia porteuse ;

Sherkha : coupure, séparation. Au Jérid, sharakha : découper en deux quelque chose d'important comme un tronc de palmier;

Smat' : au Jérid, outre (sans poils) ; rang, rangée ;

Sot'r : ligne ; 
C'est la proximité spatiale qui constitue le facteur décisif en matière de circulation des techniques, circulation repérable à travers les lexiques de l'agriculture. Ainsi que nous l'avons constaté, l'influence de Tozeur est visible dans la palmeraie proche de Castilia ; Hazoua est l'oasis la plus occidentale de la région et le lexique qui y est employé est celui en usage dans la vieille oasis de Nefta, qui lui est proche géographiquement et avec laquelle les habitants entretiennent des relations étroites ${ }^{18}$. À Dghoumes, le vocabulaire des espaces du jardin est celui des palmeraies voisines d'al-Oudiane. Des agriculteurs disent l'avoir appris, avec les techniques, dans la palmeraie d'État voisine (SODAD) où certains travaillent et côtoient d'autres jardiniers d'al-Oudiane (Degache).

Il faut noter que la diversité des dénominations et des formes ne renvoie pas à des pratiques fondamentalement différentes. Les procédés de base demeurent les mêmes. En particulier, la technique d'irrigation fait partie du bagage minimum du savoir en agriculture oasienne. Les bédouins qui passent à l'agriculture de palmeraie doivent apprendre à maîtriser cette technique. Ce n'est pas toujours le cas, comme dans les nouveaux périmètres du Nefzaoua, l'autre région tunisienne des dattes, où les parcelles sont souvent irriguées selon une logique simplifiée de deux cuvettes d'irrigation d'un seul tenant, séparées par une séguia. La notion d'irrigation sélective perd ainsi de sa pertinence dans ces contextes oasiens.

Le jar constitue l'unité géographique la plus englobante à l'échelle de la palmeraie. Il s'inscrit dans un cycle long de mouvement renvoyant à l'histoire lointaine de la région. Ainsi, la légende veut que Dghoumes soit située sur l'emplacement du royaume de Taqius que fuirent les « sept dormants " réfugiés dans une des grottes de la montagne environnante ${ }^{19}$. Les vestiges $d$ 'anciennes implantations humaines sur le piedmont de ces collines semblent conforter ces récits aux yeux des autochtones. Parfois, ce sont les traces du ruissellement des eaux sur la roche tendre qui sont identifiées à une mystérieuse écriture produite par cette civilisation disparue. Nombre de figures coraniques, telles celles de Pharaon ou de Noé, sont incorporés aux récits locaux dans une indétermination historique renvoyant aux temps premiers ${ }^{20}$.

C'est à cette échelle la rencontre d'une histoire mythique, celle de la création et d'un espace dont les contours connaissent un rythme extrêmement lent de fluctuations. Ces contours sont pourtant susceptibles d'évolutions, même si elles sont difficilement discernables en une génération. Cependant chacun peut constater leur

18 Ces relations sont toutefois limitées aux échanges de travail : du point de vue de l'agriculture d'oasis, les éleveurs Ghrib sédentarisés à Hazoua ont pu travailler dans l'oasis de $\mathrm{Nefta}$; tandis que des khammes de Nefta ont été engagés dans les palmeraies des Ghrib.

${ }^{19}$ L'histoire des « sept dormants " s'intitule en fait «La caverne », Sourate XVIII. Dghoumes n'est qu'un exemple au Jérid de la localisation par ses habitants de cette légende populaire du Moyen-Orient et coranique.

20 Sur ce point cf. Dakhlia, 1990: Chapitre 1, «Une histoire liminaire, le temps de l'ignorance ". 
réalité en suivant les traces d'anciennes cultures et en imaginant une palmeraie ininterrompue de Tozeur à Nefta comme le rapportent de nombreux récits. À l'imprécision des temps correspond l'indétermination des contours.

\section{Les jardins}

Cette échelle est celle des grands ensembles de jardins qui donnent à l'oasis la plupart de ses toponymes correspondant à un maillage intermédiaire de l'espace (entre les grands quartiers d'oasis et les parcelles privées). La plupart de ces noms sont issus d'une histoire parfois lointaine, renvoyant à un peuplement berbère par exemple, ou d'une histoire plus récente, tels les noms de jardins à Tozeur issus du démantèlement des propriétés coloniales.

\section{NOMMER LES JARDINS}

Le terme générique de jardin se traduit par ghâba qui signifie pourtant " forêt » en arabe classique (ici, on ne parle pas de ghâba pour la palmeraie, ce n'est pas « une forêt de palmiers »). Ce terme, ainsi que celui de sânya, associé à un nom constitue un toponyme, mais utilisé comme un nom commun il désigne la parcelle. Ainsi, lorsqu'on parle simplement de ghâba, c'est son jardin personnel que l'on vise : nemshi-l-ghâba (je vais au jardin); yekhdem fi-l-ghâba (il travaille au jardin) ; 'andi ghâba (j'ai un jardin). Ces noms de jardin, dont nous livrons ici quelques exemples pris ici et là, restent relativement stables dans le temps et imperméables aux restructurations foncières permanentes des palmeraies.

Une petite parcelle se dit drijât, c'est-à-dire qu'elle a la dimension de quelques darja (planches d'irrigation). Bustan désigne un beau jardin de petite taille qui a bénéficié de beaucoup d'eau. Ce terme est peu utilisé comme nom commun, davantage comme composant d'un nom de jardin. On le retrouve aussi par exemple dans al-Basâtîn (pluriel de bustan), toponyme d'une partie de la palmeraie de Nefta particulièrement bien irriguée.

Sânya (pl. swâni), quand il est utilisé comme nom commun, correspond aujourd'hui dans le Jérid à une extension plantée en jeunes palmiers et située en bord de palmeraie. Le terme désigne originellement une roue pour l'irrigation ${ }^{21}$. Mais Dozy (1927) note en Tunisie le sens de jardin donnant cet emploi curieux : tawaffa bi-saniyat bârdû, "il est décédé dans les jardins du Bardo ». En Tunisie, le terme sânya signifie actuellement un espace agricole irrigué quel qu'il soit et, principalement, les grands espaces cultivés du Nord. Son emploi pour des jardins récents en extension correspond peut-être au fait que les arbres encore jeunes ne s'élèvent pas suffisamment pour constituer un véritable ghâba. Il est peut-être aussi une manière de se démarquer de l'agriculture vieillotte du ghâba à laquelle on substitue l'image du sânya plus performant. Cette acception contemporaine du terme

${ }^{21}$ Sânya plur. swânî, in Kazimirski, 1860 ; Dozy, 1927. 
ne doit cependant pas nous faire oublier qu'il est à l'origine de nombreux noms d'anciennes grandes propriétés ${ }^{22}$.

Les jardins d'extension dénotent, en effet, un esprit d'entreprise comparable à celui qui anime certains agriculteurs de la région voisine du Nefzaoua (la première région dattière de Tunisie) qui exploitent plus de 6000 ha de périmètres irrigués par des sources privées obtenues par forages dans la nappe phréatique dite «Complexe terminal $»^{23}$.

Un autre mot du vocabulaire local dérive du français: numro (plur. nwâmir). Ce sont les jardins des palmeraies nouvelles loties en parcelles numérotées. Conçus par les autorités coloniales (ou les colons eux-mêmes), puis par l'État tunisien, les lots ont été alloués pendant la période récente aux (dits) anciens combattants et à des nomades dans le cadre des programmes de sédentarisation (oasis de Chemsa, Hazoua, Dghoumes...). Ces jardins d'anciens nomades ont une vocation agricole plus marquée et leurs processus de socialisation sont différents de ceux des palmeraies de vieille tradition oasienne.

\section{LE FONCIER}

La permanence des noms de jardin ne doit pas laisser penser que la structuration foncière de la palmeraie est immuable. À travers les générations, une mobilité des propriétés existe. Nous assistons aussi à la fin d'une gestion de l'oasis par le lignage et du patrimoine par la famille élargie. Il est difficile de s'avancer sur ce qu'était la gestion des terres autrefois au-delà d'une tradition idéale fondée sur la gestion de l'héritage dans l'oasis par le fils aîné, mais aujourd'hui elle s'identifie à un double mouvement : l'indivision et le morcellement.

L'indivision de nos jours n'est plus guère le résultat d'une stratégie, mais elle résulte d'un non-accord sur la répartition de l'héritage. Au mieux, seul un minimum d'investissement est pratiqué, sinon le défaut d'entretien déprécie à terme la valeur du jardin. Le morcellement décompose le jardin en autant d'unités que d'héritiers, diminuant la rentabilité et la possibilité d'investissement (voir document 4: le jardin A a d'abord été morcelé, puis une partie maintenue en indivision par défaut, car le terrain était devenu trop exigu, et est plutôt aujourd'hui un jardin d'agrément ; l'indivision du jardin $B$ de grande superficie ressemble davantage à une stratégie de maintien du capital paternel doublé d'un désintérêt personnel des frères pour l'agriculture).

Les oasiens mentionnent qu'autrefois (bikri) se manifestait une réticence à la vente d'une terre héritée. Ce n'est globalement plus le cas aujourd'hui, même si la propriété conserve son importance affective et symbolique. Dans l'oasis, il n'existe pas d'unité minimale de transaction. L'anecdote du palmier appartenant à plusieurs personnes se retrouve dans plusieurs oasis sahariennes. Jusqu'à l'indépendance, les

\footnotetext{
${ }^{22}$ Par exemple sânyat rah'îl à Nefta, sânyat al-bir à Tozeur ou sânyat fayyal à Degache.

${ }^{23}$ Une estimation du CRDA de Kébili fait état en 1998 de 6059 ha. Pour comparaison, la palmeraie de Nefta représente environ 850 ha et celle de Tozeur environ 1000 ha.
} 
différents éléments de la propriété (eau, terre, palmier) pouvaient faire l'objet de transactions séparées. C'est pour cela que le législateur a dû inventer un terme désignant la propriété totale d'une parcelle dans la palmeraie, le jidar(Hénia, 1980).

1 - Jardin A - Acquisition en 1880 par achat : 2 hectares et demi

A h m ed

il laisse 3 parts à sa mort

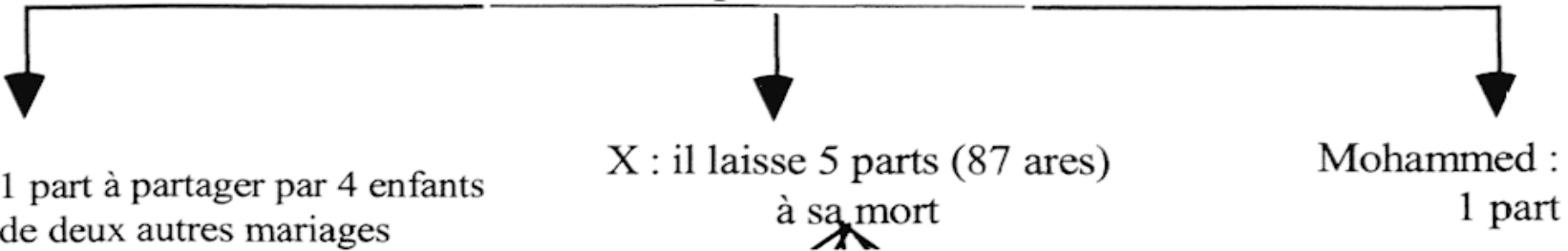

(ils reçoivent des parts dans d'autres jardins)

Superficie totale :

87 ares en indivision
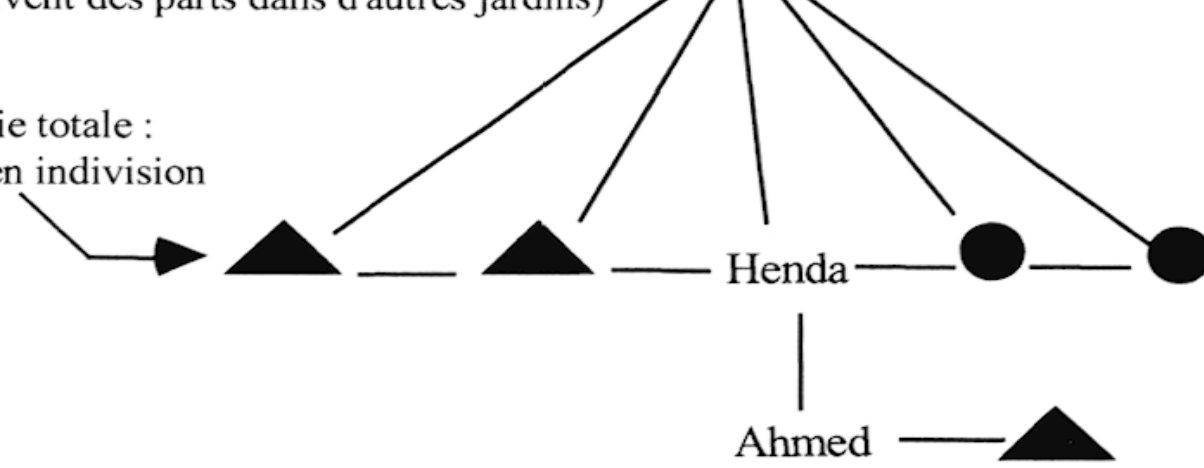

Gestion en indivision des cinq parts par

Ahmed (fils de Henda) et partage des

recettes ; 1 khammes effectue le travail. 


\section{2 - Jardin B - Palmiers plantés en 1922-1923; 7 hectares}

el-Soudanî, le concepteur du jardin, souscrit un crédit auprès du colon Binet

$$
\downarrow
$$

Binet récupère le jardin de Soudanî incapable de payer ses dettes<smiles>CCOC</smiles>

L'Etat récupère à l'Indépendance le jardin et le met en vente avec facilité de paiement

Mohmoud se porte acquéreur et laisse 6 successeurs mâles qui reçoivent chacun une part

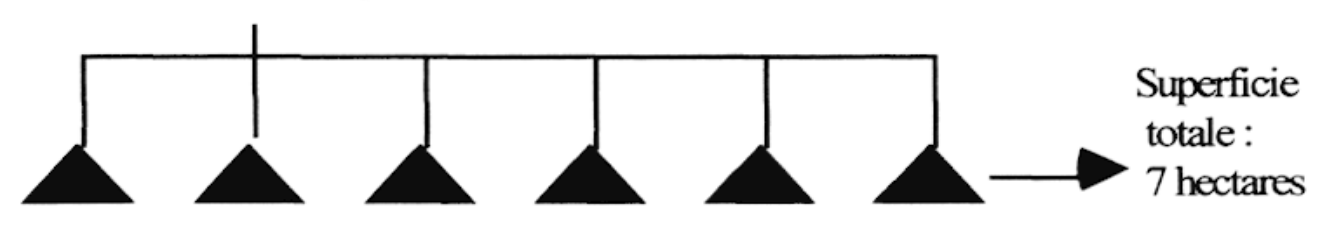

Gestion en indivision des 7 parts, par un des fils qui travaille avec l'aide de journaliers ; partage des recettes.

Document 4 : Deux exemples d'évolution de la structure foncière

On trouve ainsi jusqu'au $\mathrm{XIX}^{\mathbf{c}}$ siècle dans les actes notariés la mention de ce mot jidar, qui désignait par ailleurs dans le parler local un petit mur en terre servant à retenir l'eau.

Les biens indivisibles ont pris fin avec la disparition des biens habus. Ceuxci, quand ils sont de type familial $a h l^{24}$ « prévoient que les biens d'une famille seraient dévolus à une fondation pieuse en cas d'extinction de la descendance mâle " (Bédoucha, 1987 : 87). Dans les oasis du Jérid, leur dissolution par l'État tunisien à

${ }^{24}$ On distingue trois types de biens habous : ahlî, le bien reste dans la famille jusqu'à son extinction où il revient alors à une fondation pieuse; khayri, il est attribué à une œuvre de bienfaisance et zawiya, il est attribué à l'entretien d'une fondation pieuse. 
l'indépendance a été à l'origine d'un mouvement foncier facilitant l'entrée de nouveaux propriétaires, dont certains de souche nomade.

La disparité des situations et des conceptions de la propriété oasienne implique une diversité des stratégies. Certaines tendent à la reconstitution de grandes parcelles d'un seul tenant, telles qu'on les présentait autrefois. Ainsi, on divise, on rachète des parts, on revend, on cède, on échange. La structure foncière se présente comme un puzzle mouvant au sein de l'oasis.

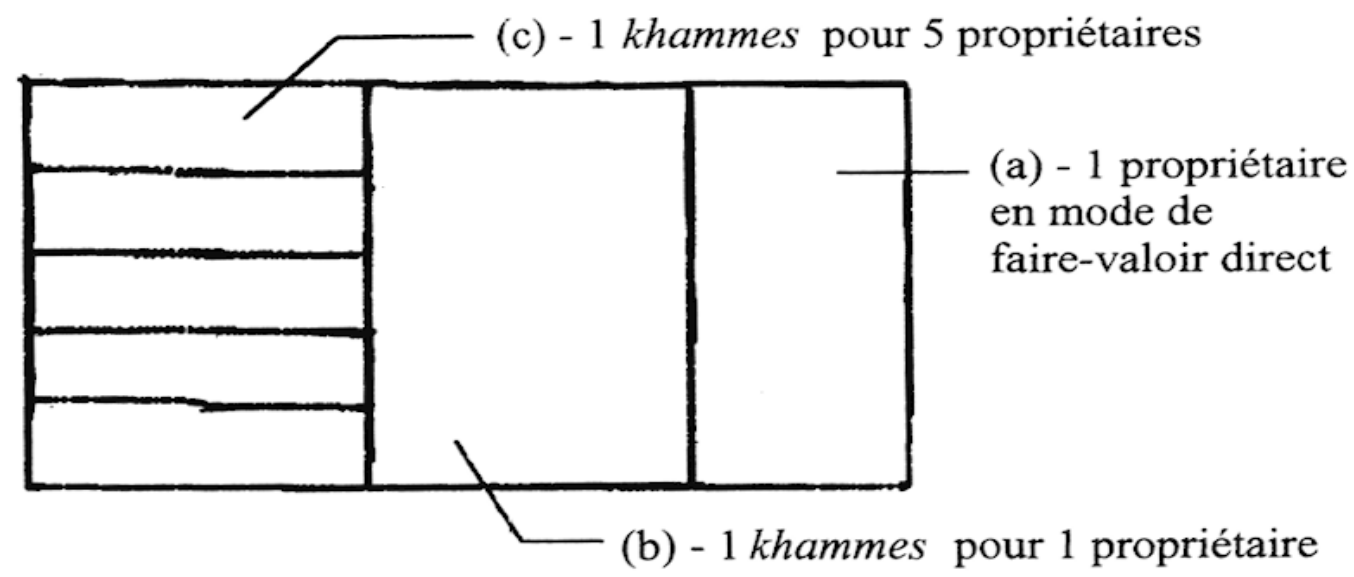
Document 5 : Structure foncière de Sânyat Rah'îl à Nefta (la forme du jardin est
schématisée)

L'ensemble du jardin (document 5) de Sânyat Rah'îl a appartenu à un Juif. Il y a 80 ans, dit-on, il l'aurait vendu au grand-père d'un des propriétaires qui y possède aujourd'hui 0,7 ha qu'il travaille lui-même (a). Deux khammes (métayers au cinquième) exploitent le reste du jardin, l'un pour le compte d'un propriétaire (b) et l'autre sur une surface sensiblement identique, mais divisée en cinq propriétés distinctes (c).

L'oasis se compose de milliers de jardins. Par le biais des transactions, les agriculteurs ont une emprise sur la palmeraie, lui donnent son visage et des noms pour la décrire; les différentes dénominations du jardin renvoient souvent à des conceptions différentes de l'agriculture. À ce niveau d'organisation de l'oasis, de maîtrise de la terre correspond le temps moyen des décennies, des générations au cours desquelles se mettent en place les noms de jardins.

\section{DISPOSITION DE LA PARCELLE}

Dans la région du Jérid, la répartition des palmiers à l'intérieur du jardin est homogène. Dans les oasis de Gabès sur le littoral, les palmiers ont plus une vocation de coupe-vent que de production (en raison d'une mauvaise fructification due au climat maritime) et ils sont disposés en haie autour de grandes planches de cultures. 
La disposition homogène au Jérid induit pour une part la forte densité des pieds à 1'hectare. Ce rapport atteint parfois 400 palmiers par hectare dans les vieilles oasis (les pieds sont alors en désordre) contre 100 par hectare (les palmiers sont en ordre aligné) dans les nouvelles plantations.

Verticalement, la structuration en trois strates est connue et demeure d'actualité dans un grand nombre de jardins de vieilles oasis. Sous l'étage des palmiers poussent les arbres fruitiers et le troisième étage, à l'ombre, est celui des cultures maraîchères et fourragères. Celles-ci sont contenues dans les planches de cultures. Ces planches répondent à une logique géométrique d'imbrication et d'emboîtement de quadrilatères.

Horizontalement, deux zones se distinguent dans le jardin : celle de la production agricole et celle de l'espace non cultivé où se trouve la cabane (document 6, et comparer avec le document 7 un peu plus loin : Plan d'un jardin en palmeraie récente). Celle-ci peut être nommée grîsha (enclos, hutte) ou “arish. Une fois passée la porte (bâb), l'allée principale (al-ribat-al-asâsiya) nous y conduit directement. Là, les braises du feu sont toujours actives. La cabane contient des outils de jardinage et parfois un lit qui permet la présence du jardinier, outre pendant les siestes, la nuit lors des irrigations par exemple ${ }^{25}$. Dans quelques jardins existe un abri pour les animaux d'élevage. Cet abri se dit kûri du mot français écurie, bien qu'il corresponde plutôt à étable ou bergerie. Les animaux qui ne sont pas dans les pâturages sont plus fréquemment proches de la maison.

Le système de disposition des cultures en planches d'irrigation est très commun aux oasis sahariennes. Au sol dans les jardins, la surface cultivée et irriguée est délimitée en planches. Au Jérid, l'opération du tracé est appelée tafs'îl, nom d'action du verbe fass'ala, « détailler ». Ainsi l'opération consistant à constituer l'ordonnancement des cuvettes et des séguias est-elle placée sous le sceau de la minutie. C'est bien d'une mise en détail qu'il s'agit, rappelant par exemple cette «mise en carreau sous les pieds des hommes » des jardins dogons : «damiers réguliers de carrés formés par des diguettes de moins d'une palme de large ». Ce n'est pas ici le lieu de s'arrêter sur l'importance symbolique que revêtent chacune de ses opérations apparemment purement techniques, mais les manières de faire de paysans ou d'artisans à travers le monde ont aussi souvent cette dimension « à dire le monde » (Griaule, 1985 [1 $1^{\text {re }}$ éd. 1948] : 72).

25 ...bien qu'il soit très rare que l'on passe la nuit entière dans le jardin, cela étant plutôt le fait de personnes marginalisées vivant sur leur parcelle (par exemple de vieux célibataires). 


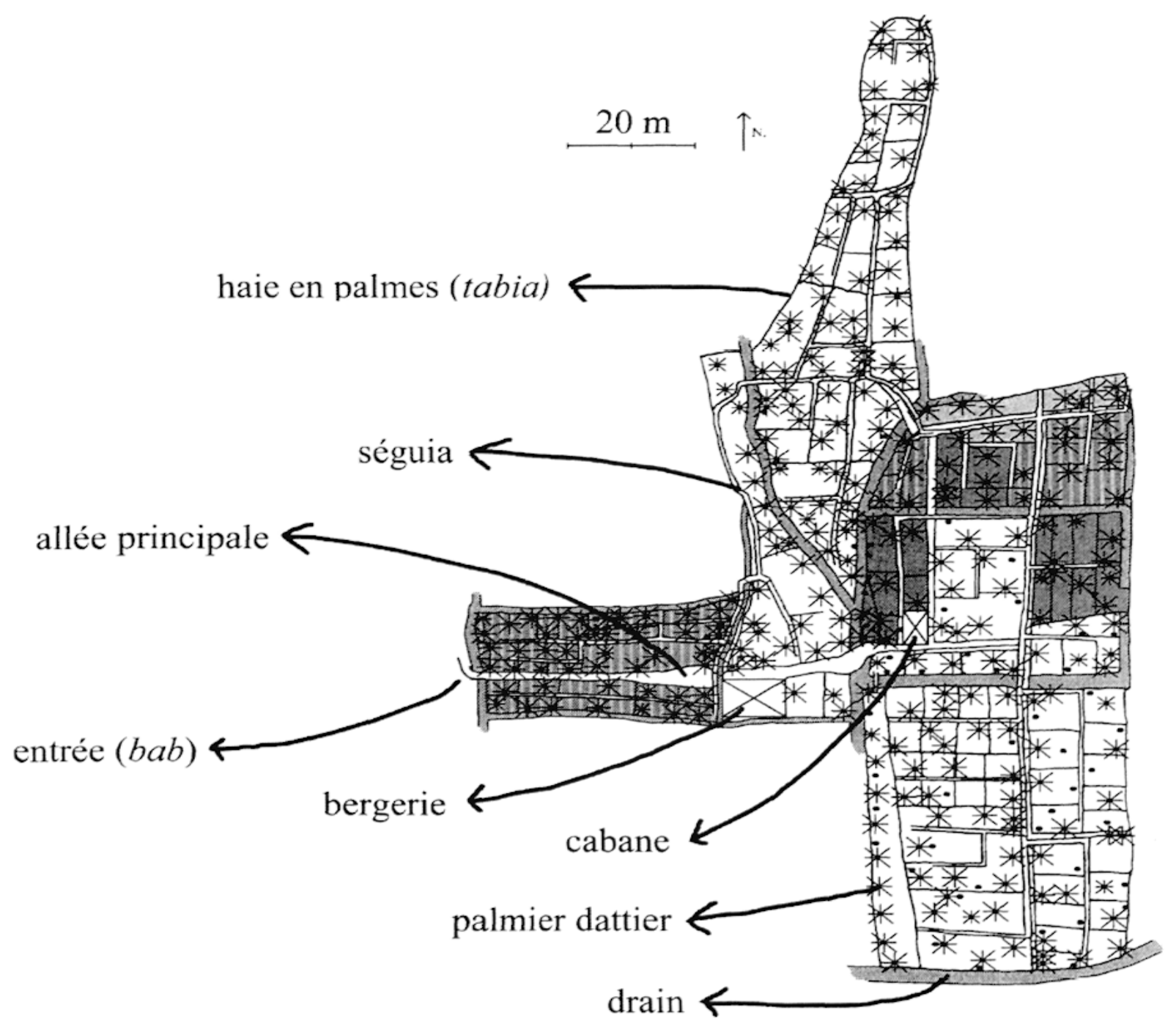

Document 6 : Plan d'un jardin de vieille palmeraie (à al-Hamma) (Source : Battesti, 1998, modifié)

L'espace de la parcelle se définit dans sa forme sur quelques années. L'opération d'aménagement des cuvettes (tafs'îl) est, en effet, rééditée tous les quatre ans, la période du cycle de labour profond (un quart de la parcelle par an en théorie, mais en fait un cycle peut durer jusqu'à six ou sept ans en vieille palmeraie et s'écourter à deux ans en jardin de création récente). L'agriculteur décide seul de l'organisation de ses planches de cultures. Il possède «le dessin dans la tête " puis l'applique et le confronte à la réalité, l'adapte à la forme du jardin, d'où peut-être aussi la diversité des formes et des noms en anciennes oasis sur un terrain accidenté et travaillé par l'histoire. 
C'est là une échelle complètement maîtrisée individuellement dont la temporalité peut être appréhendée à l'échelle d'une vie. Le tafs 'îl encadre une partie de la temporalité de la parcelle, l'autre étant définie par le calendrier des activités purement culturales comme la cueillette annuelle des dattes ou les deux saisons annuelles de production maraîchère (les agriculteurs distinguent les plantes estivales, $s$ 'eyfi et les plantes hivernales, shitawi) ${ }^{26}$.

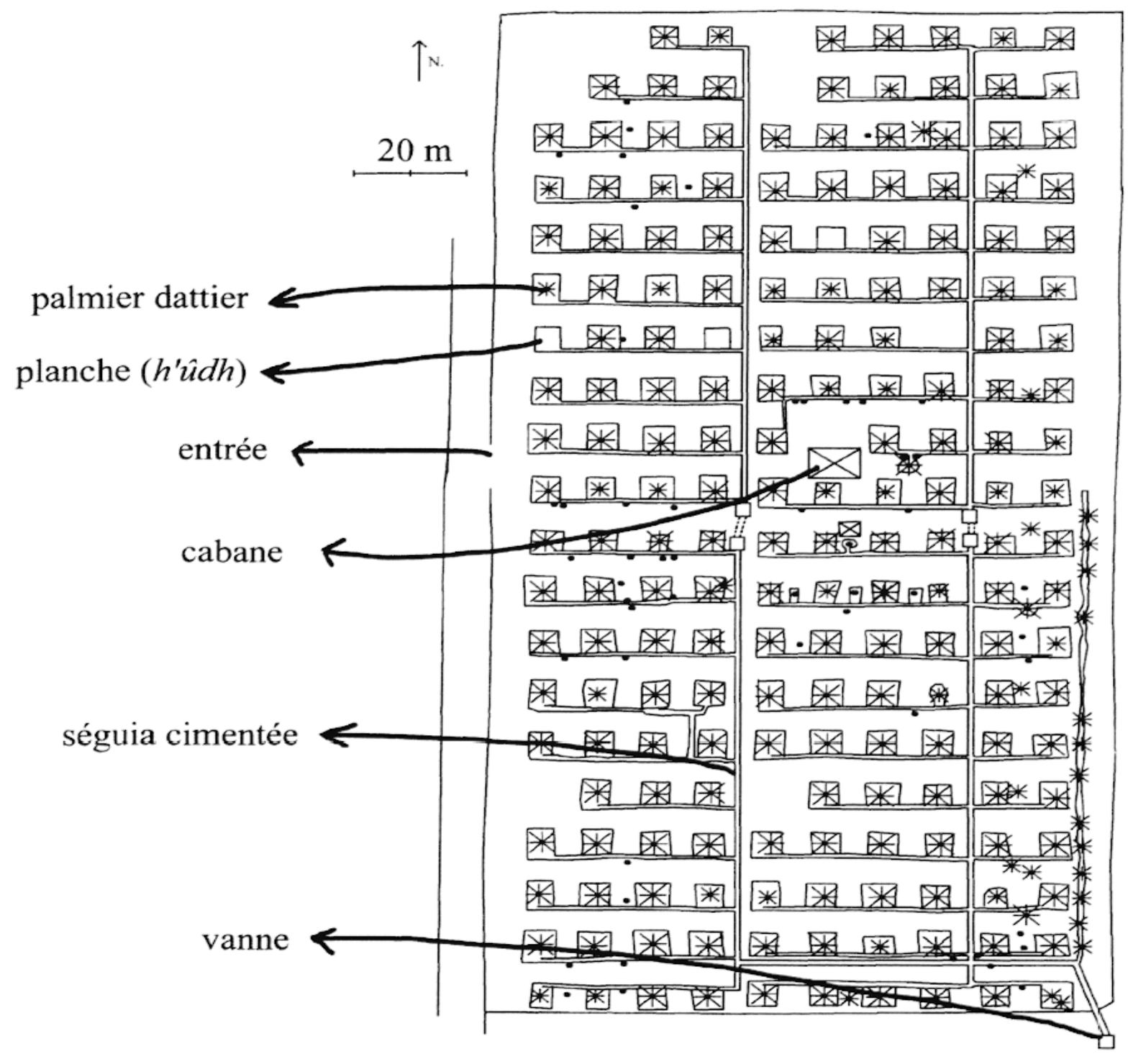

Document 7 : Plan d'un jardin de palmeraie récente (à Ibn Chabbat) (Source : d'après V. Battesti, 1998)

${ }^{26}$ Cf. sur ce point Battesti, 1998. 
Les oasis partagent une nécessaire gestion de l'eau qui détermine, partiellement, la disposition intérieure des parcelles. Il convient au moins d'amener l'eau aux palmiers dattiers et le cas échéant aux cultures basses. Par ce système de planches, il est possible aussi d'irriguer de manière différentielle selon la présence ou non de cultures et selon leurs besoins physiologiques. Il en résulte au sol un dessin complexe de swâgi (pluriel de segya) et de planches de cultures.

L'intérieur du jardin est le niveau de l'emprise sur la terre non de la génération, mais cette fois de l'individu. C'est là le niveau du travail sur le sol, de la détermination des formes du tafs'îl, l'échelle où le jardinier peut agir de manière directe sur les choses. C'est l'espace restreint mais révisable, le niveau du temps court où l'action est la plus immédiate.

Dans ces espaces oasiens fortement anthropisés, le maillage de l'espace correspond à une logique d'emboîtement et à chaque échelle spatiale (petite, moyenne, grande) correspond une temporalité propre. Afin de juger de la qualité des lieux de la palmeraie, il demeure nécessaire de saisir les pratiques de ces espaces : comment l'espace est-il approprié, comment est-il transformé en lieu ?

\section{PRATIQUES DE L'ESPACE : DE L'ESPACE AU LIEU}

M. Augé commentant $M$. de Certeau remarque qu'il fait de l'espace un « lieu pratiqué » opposant «le lieu à l'espace comme la figure géométrique au mouvement, le mot tu au mot parlé, ou l'état au parcours » (Augé, 1992 : 104). Cette opposition que nous adoptons, sinon que nous inversons espace et lieu, est une invitation à prendre en compte les usages de l'espace. Le lieu est l'espace pratiqué : «l'usage est producteur d'espace, ou, plutôt de lieux, de territoires, c'est-à-dire d'espaces qualifiés, féminins ou masculins, appropriés par des dénominations, des utilisations, des représentations, des fréquentations, un entretien ou une absence d'entretien... » (Depaule, 1995 : 27).

Le premier groupe d'acteurs venant à l'esprit est constitué des oasiens dans leur ghâba qui donnent sens à l'espace par leurs pratiques quotidiennes, solitaires ou collectives. Mais les femmes et les touristes également s'approprient ces espaces.

\section{Travaux et plaisirs solitaires}

Le khammes (métayer), ou le propriétaire quand l'exploitation est en mode de faire-valoir direct, passe la journée complète au jardin, à moins qu'il n'exerce une pluri-activité au niveau familial ou individuel, système qui permet souvent le maintien de l'activité oasienne. Le temps passé dans le jardin est très souvent solitaire, l'hiver environ de 8 à 16 heures et l'été de 5 ou 6 heures à midi, plus rarement l'après-midi. Le jardinier demeure seul à s'occuper des cultures. Un temps loin d'être négligeable est consacré au nettoyage de la parcelle, une réelle préoccupation esthétique est confirmée par la présence de fleurs cultivées (jasmin, rose...) et d'arbres ornementaux. 
Selon ces représentations, s'effectue sur le beau ${ }^{27}$ un travail qui n'est pas toujours spécifique, car l'esthétique peut ressortir d'activités ordinaires : les dessins du tafs'îl, la terre mise à nue, la disposition de semis de fèves en pourtour de planches cultivées... Cette pratique quasi épicurienne du jardin renvoie à une " esthétique fonctionnelle » dans laquelle « le sentiment esthétique réside dans la relation satisfaisante entre le sujet et le monde qui l'entoure » (Leroi-Gourhan, $1956: 1)$.

Le travail ne couvre pas la totalité de la journée; assis ou allongé, cigarettes, siestes, repas, thés bien sûr, en occupent une partie non négligeable. « Dans l'oasis, il [le jardinier] se sent protégé, dans le calme et humide retrait clos d'un lieu fermé, à l'abri du monde " (Duvignaud, $1992: 52$ ). Loin du village dans cet espace privé, clos d'une haie de palmes sèches (zarab à Tozeur ou t'abya à Degache) ou d'une frontière ( $h$ 'ad) marquée par une buttée de terre, un espace quasi intime défendu quelquefois par des ossements d'animaux (souvent un crâne) fixés à un arbre comme un paratonnerre contre le mauvais œil. Le jardin est plus qu'un simple lieu de travail ou qu'un lieu à vocation purement agricole; il est commun d'y rester même si la nécessité de travaux ne se fait pas sentir. Le jardinier en quelque sorte habite son jardin, $y$ vit dans la journée et la soirée pendant les saisons chaudes en dehors d'un temps précis. Les jours se précisent quand on emploie des salariés à la journée, les heures au moment de la nûba (tour d'eau), mais pas tant pour l'heure qu'il est (on règle sa montre sur celle de son voisin) que pour s'accorder sur une durée comme c'était le cas avec l'ancien gaddus ${ }^{28}$.

Le jardin de vieille oasis est donc pour le jardinier, à la fois un espace de production agricole, mais selon des critères qui ne tiennent pas toujours de la pure productivité économique ${ }^{29}$, et un espace socialisé échappant à la logique productive. Il s'agit d'un lieu clos et intime, dont l'esthétique s'expose au regard des autres, apprécié ou jalousé (ce qui est source de mauvais œil). Car le jardin possède aussi une certaine dimension collective.

\section{Le collectif dans le jardin}

L'accueil et le devoir d'hospitalité dans le jardin sont systématiques. Parfois le partage du repas mais surtout du thé sont l'occasion d'échanges, de discussions. Certains jardins accueillent les buveurs de legmi (sève de palmier), qui est souvent en fait du qêsham (legmi fermenté). Les participants à ces «club legmi» (parfois payants) peuvent provenir d'un cercle débordant la palmeraie (fonctionnaires, commerçants...). Implicitement, les bienfaits que procure la parcelle renvoient à l'idée de janna (paradis): on y prélève légumes, fruits, boisson fermentée et

\footnotetext{
27 « Regarde comme il est beau ce jardin », nous ont souvent dit les jardiniers.

${ }^{28}$ Il s'agit de l'ancienne unité de temps utilisée pour l'irrigation et matérialisée dans la durée que met l'eau à s'écouler du trou du godet en poterie.

${ }^{29}$ Ainsi en est-il du maintien actif (on plante encore) de cultivars " non-rentables " d'un point de vue économique (par rapport à la deglet nour, voire à l'allig) ou de palmiers dits shkan et khalt qui participent à une biodiversité in situ.
} 
autrefois le takruri (cannabis), un idéal d'abondance correspondant à l'eschatologie musulmane. Être bien, c'est apprécier le lieu, un confort visuel, olfactif; bien ensemble, dans la consommation, la communication (les discussions, les poésies et les chants). Cet esprit n'est pas l'apanage du Jérid, et on le retrouve par exemple « dans la culture japonaise, [où] l'appréciation des beaux sites est indissociable du commerce humain (échange de poèmes, banquets, cérémonies du thé) » selon $A$. Berque. Entre l'esthétique du jardinier jridî et celle du touriste occidental trouve-t-on peut-être l'opposition qu'Augustin Berque (1993: 33) fait entre «le paysage sociable » et le «paysage contemporain solitaire » inspiré de l'Occident romantique (le touriste et le coucher de soleil par exemple, en particulier sur le site du « belvédère » à Tozeur).

Dans l'oasis fleurissent toujours ce que Duvignaud nomme les « parleries » : h'alga (cercle) et surtout, $g a^{c} d a$ (du verbe dialectal ga $a d$, s'asseoir et qui désigne une réunion masculine), occasions d'échanges des savoirs. Les normes esthétiques se communiquent, le collectif sanctionne l'individuel, les connaissances se transmettent, des recettes, des essais, des commentaires sont échangés ; on écoute, on se souvient de récits, d'histoires locales. Une partie de l'existence collective se joue là.

La production agricole nécessite aussi cette intervention du collectif dans le cadre de l'entraide ou du salariat. Et ceci particulièrement autour du palmier dattier dont le calendrier des activités rythme l'année : pollinisation au printemps (d.ukar), suspension des régimes au début de l'été (imferza) et récolte à l'automne. Mais si deux ou trois personnes peuvent s'acquitter des deux premières tâches, la récolte est faite par des équipes nombreuses. À cette occasion, les palmeraies résonnent des chants des ouvriers qu'orchestre souvent le gatta $^{c}$, le coupeur ${ }^{30}$. Ces ambiances sonores, comme celles des soirées de printemps alors que s'élèvent des jardins chants et percussions, rires et éclats de voix, constituent une des structurations de cet espace, certes plus volatile et donc moins visible que les trajets par exemple, mais non moins importante.

Le jardin est donc tout à la fois un lieu intime et protégé et un lieu de partage ouvert à la visite, tout à la fois un lieu de solitude et de sociabilité forte dans lequel le travail n'est pas la seule préoccupation. La notion de travail ne recoupe que partiellement celle de l'agronome. Le ghâba représente un lieu de communication, où s'échangent et s'éprouvent des normes techniques et sociales.

\section{Représentations de la palmeraie}

Geneviève Bédoucha, dans son ouvrage L'eau, l'ami du puissant, retient la racine $j-r-a$ comme base du mot jar. Cette racine signifie courir, mais aussi ruisseler

${ }^{30}$ Le gatta (de gata ${ }^{c}$, couper) coupe le régime et le tend aux meddad-s (de madda, tendre) qui alignés le long du stipe se le passent jusqu'à ce qu'il atteigne le sol. Le gatta qui officie le plus haut et dont le travail requiert une technicité plus élaborée est généralement le mieux payé de l'équipe des collecteurs et bénéficie d'un plus grand prestige. 
et s'écouler. Ainsi, le terme jar a signifié dans les oasis (du Nefzaoua tout au moins) le temps de parcours de l'eau dans les jardins puis, par extension, la palmeraie dans son ensemble. Le pluriel jrûr n'est employé que dans le sens de terroirs, quartiers d'oasis. Cette étymologie recueillie par Bédoucha (1987: 16) dans la région du Nefzaoua témoigne d'un système dans lequel «l'eau est première techniquement et économiquement, mais aussi symboliquement ». De même, M. Rouissi (1973 : 83) indique qu'à Degache, le terme jar renvoie, en même temps qu'à un quartier d'oasis, à « ce qui reste d'eau dans une rigole lorsque le tour d'eau est terminé ».

Dans le Jérid actuel, une autre étymologie est proposée. Certains, en effet, voient dans le terme jar un dérivé du terme jâr, voisin. Cette étymologie exprime l'aspect «puzzle » de la palmeraie conçue sous l'angle de sa morphologie foncière : d'un voisin à l'autre, min aj-jâr li-l-jâr.

Dans ce contexte, l'eau est éludée comme interprétation alors qu'elle demeure bien sûr tout aussi nécessaire d'un point de vue écologique. Ceci pourrait s'expliquer par le fait que la gestion de l'eau échappe aux agriculteurs ${ }^{31}$, et qu'ils sont dans ce domaine désormais cantonnés à un registre revendicatif ${ }^{32}$. De fait, la fin de la gestion collective et locale de l'eau a conduit à un changement dans l'importance relative des facteurs de production entraînant une fluidité dans les représentations. Désormais, la propriété de la terre et de l'eau sont indissociables (sauf arrangements illicites). Ainsi, les superficies peuvent être exprimées en temps d'eau $^{33}$, ce qui dans un système où l'eau s'échangeait indépendamment de la terre n'avait pas de sens.

La dépossession de la gestion collective et locale de l'eau, à la suite de la prise en charge des forages par l'État et consécutivement de la disparition de l'artésianisme, a fortement réduit la part des travaux collectifs dans la palmeraie. Les travaux par les khammesa de nettoyage des abords, de curage des drains et des oueds pour maintenir le débit ont disparu; ce sont les services de l'agriculture qui s'en chargent désormais.

${ }^{31}$ Cette gestion revient aujourd'hui à l'État qui la sous-traite cependant aux AIC qui sont chargées de la distribution et de la commercialisation. Mais, bien souvent, ces associations échappent à l'immense majorité des agriculteurs et ce sont de riches propriétaires qui s'y imposent.

32 Il est à noter que dans le Sahel tunisien, on parle de mjar, qui est le lieu aménagé pour permettre au animaux de s'abreuver. Le terme est un nom de lieu issu de la racine $j-r-r$ qui signifie tirer. C'est encore une possibilité pour l'étymologie du mot jar, dont la première consonne aurait été assimilée et qui serait donc issue d'un champ sémantique désignant l'endroit où l'on tire - et par extension amène - l'eau. De même, le géographe A. Kassah (1996) écrit dans son ouvrage "wahât al-Jérid», chjar, qui renvoie ainsi orthographié au collectif du terme « arbre ».

33 La superficie en ares d'un jardin peut être déduite de son temps d'eau (nûba), par le truchement d'un coefficient lié au débit. Par exemple, dans la partie Beni Ali de Nefta, ce coefficient est de 1,8. Ainsi, un jardin qui a deux heures et vingt minutes d'eau mesure $140 / 1,8=$ environ 77 ares. 
Des logiques différentes voient le jour : celle des responsables étatiques de l'agriculture par exemple qui veulent cimenter les oueds pour qu'il y ait moins de déperdition d'eau, mais aussi celle des touristes et de leurs guides pour lesquels un oued cimenté est moins beau, moins "couleur locale » qu'un oued sableux. Les visiteurs étrangers, ou originaires des villes du Nord et du Sahel sont en quête de traditionnel et d'authenticité que le Sud incarne à leurs yeux. Aux aménageurs de tenir compte des attentes de chacun, des nécessités de la production agricole et de celles inhérentes à la vente d'un produit touristique axé sur une image figée du désert et des oasis. Certains jeunes de Tozeur, d'ailleurs peu intéressés par l'agriculture, peuvent être influencés par les visions de l'oasis comme objet de consommation touristique, ou du moins la prendre en compte, voire l'adopter partiellement.

\section{Trajets}

Les trajets des touristes sont bien définis dans les centres d'intérêt touristique que sont Tozeur et Nefta. La route suivie, dans le cas de Tozeur, décrit une boucle dans la palmeraie. Elle est goudronnée et, par endroit, des réverbères l'éclairent dans la nuit. Le parking des calèches attenant à la palmeraie héberge une petite foule de marchands qui attendent de pied ferme les cars de visiteurs. Le parcours et ses étapes aux noms révélateurs (le Zoo du désert, le Jardin du paradis, le Belvédère...) propose une version correspondant aux imaginaires occidentaux construits sur la luxuriance de l'oasis opposée aux dunes arides du Sahara. Cette conception peut être partagée par les Tunisiens du Nord et du Sahel qui connaissent souvent très mal le Sud du pays.

La plupart des touristes, en majorité français et allemands, oppose la palmeraie perçue comme un paysage naturel, en quelque sorte offert aux hommes, à l'austérité menaçante du désert. Cette perception romantique d'une nature fraîche et accueillante ne recoupe pas celle des oasiens pour lesquels la palmeraie, sans arrêt domestiquée et travaillée, est issue des laborieux efforts humains. L'ordre minutieux intérieur, le nettoyage, l'arrachage des mauvaises herbes permettent de contrôler un espace dans lequel le sauvage (wih'shî) pourrait être cette marge incontrôlée qui borde l'univers ordonné. Les sangliers, les mauvaises herbes, les roseaux qui prolifèrent dans les mares stagnantes, voire les jinn-s qu'on imagine tapis dans les drains (khandig), les trous et les mares croupissantes en font partie, ainsi que les fantômes (shabah'). Le sauvage serait cette fin de 1'ordonné dès que l'attention humaine se relâche et que la nature n'est plus contrôlée et domestiquée (jardins abandonnés, oued-s enherbés, prolifération de nuisibles ...).

Pourtant les trajets apprivoisent la palmeraie, la structurent en chemins, sentiers, passages. Vers l'Aïn (source de Tozeur), désormais alimentée en eau par un forage, hommes et femmes se dirigent séparément pour se laver dans son eau brûlante. Les trajets féminins sont cependant de plus en plus rares, car désormais le touriste peut surgir à n'importe quel moment, ignorant les règles locales, troublant l'intimité des femmes. Promenade de femmes, encore, lorsqu'au bord de l'oasis, rarement plus loin, et à quelques occasions précises, elles cueillent des fleurs (pour la fête de printemps en particulier). 
Les visites aux saints disséminés dans les palmeraies du Jérid sont une autre occasion de visites féminines à l'intérieur même de la palmeraie. Jocelyne Dakhlia (1990 : 96) dans son ouvrage sur le Jérid a noté que « la palmeraie de Tozeur abrite une nébuleuse de tombes de saints (...) ; les saints, porteurs de Baraka, sont les seuls morts qui puissent être admis dans les jardins "».

Le sacré s'inscrit donc dans la palmeraie ; les mausolées constituent autant de repères d'orientation et suscitent des toponymes. La «densité de la sainteté dans les paysages oasiens » est un indicateur de leur valeur symbolique.

Le niveau le plus large d'observation, la palmeraie dans son ensemble, est celui qui appelle le plus de perceptions différentes, car il est alors pratiqué par des groupes variés. À une conception d'une domestication permanente du jardinier se superpose la vue «naturaliste » du touriste. Mais tous deux font de l'espace des lieux qu'ils qualifient par leurs pratiques. L'un quotidiennement, l'autre par la succession de son semblable sur un trajet codifié.

À ces pratiques correspondent des compétences, notamment linguistiques, des connaissances des noms, des toponymes, des localisations, une vision d'ensemble des jardins (direction et capacité de survol), des représentations imaginaires... ${ }^{34}$. Le sacré et la sociabilité, la magie et le déambulatoire participent à la qualité des lieux dans les anciennes palmeraies.

\section{POLARITÉS ESPACE/TEMPS}

Différentes polarités espaces/temps structurent les palmeraies à travers les pratiques locales. L'espace large du terroir, celui du jar, se construit dans le temps long, le millénaire. Son histoire correspond au temps des mythes d'origine, des récits de fondation. C'est le niveau le moins malléable par les individus ; l'emprise est la moins importante et la plus indirecte, les contours sont flous et l'appropriation se fait dans l'imaginaire.

L'espace intermédiaire des jardins évolue sur plusieurs décennies, à l'échelle de deux où trois générations, les oasiens ont ainsi la maîtrise de l'histoire récente des parcelles. Le rythme de changement est plus rapide. Les noms de jardins sont connus. L'action humaine s'inscrit visiblement sur le sol; les contours bien qu'irréguliers sont définis. Certains noms de jardins renvoient parfois à des histoires plus anciennes. Ils témoignent d'un passé berbère par exemple ou d'une présence juive qui, elle, n'a disparu que très récemment.

L'espace à l'intérieur du jardin se définit enfin dans sa forme sur quelques années. Le tafs' $̂ l$ est réédité tous les quatre ans en théorie (en pratique entre deux et sept ans). C'est une échelle complètement maîtrisée individuellement. Le jardin représente un espace de vie et de rêve, de travail et de sociabilité. Un savoir agraire

34 G. Bédoucha (1987) note que les femmes connaissent les jardins de la palmeraie dans l'abstrait et par rapport à l'eau sans en connaître la localisation. 
fort est nécessaire à la reproduction biologique de l'espace. Les contours sont nets et les formes internes structurées. La domestication est permanente et son résultat peut être remis en cause au moindre relâchement du travail. Cela détermine une tension dans le rapport à la terre qui définit le niveau du travail agricole.

La logique spatiale de l'emboîtement est poursuivie jusque dans le travail du sol. L'arrangement géométrique des planches participe de cette spatialité. Sa mise en cuvre nécessite une compétence importante. La diversité des lexiques renseigne sur le caractère très individualisé de chaque terroir, tandis que les rapprochements lexicaux sont des indicateurs des relations entre les agriculteurs des diverses oasis.

Il existe des logiques de traverse, de trajet auxquelles participent de façon conjoncturelle les touristes et les femmes. Celles-ci constituent une autre forme d'appropriation de l'espace qui passe par des territorialisations temporaires, davantage assujetties à des moments et à des saisons que celles des agriculteurs.

La palmeraie, objet limité et clos, facilement circonscrit a priori, se révèle un espace complexe dans lequel n'existent pas de lieux simples et objectifs soumis à une temporalité unique.

\section{BIBLIOGRAPHIE}

AUGÉ M., 1992.- Non-lieux, Introduction à une anthropologie de la surmodernité, Paris, Seuil.

BATTESTI V., 1998.- Les relations équivoques, Approches circonspectes pour une socioécologie des oasis sahariennes, th. Anthropologie sociale, Uni. Paris V-MNHN.

BÉdOUCHA G., 1987.- L'eau l'ami du puissant, Une communauté oasienne du Sud tunisien, Paris, Éditions des archives contemporaines.

BERQUe A., 1993.- Du geste à la cité, Formes urbaines et lien social au Japon, Paris, Gallimard.

Bisson J., 1995.- Les marges sahariennes: lieux d'affrontement des spatialités, Actes du Séminaire "Les oasis au Maghreb, Mise en valeur et développement", Gabès 4, 5 et 6 Nov. 1994, Tunis, Cahier du Ceres, 12, 13-28.

CONDOMINAS G., 1980.- Introduction: L'espace social, in Condominas G., dir., L'espace social, À propos de l'Asie du Sud-Est, Paris, Flammarion : 11-94.

DAKHLIA J., 1990.- L'oubli de la cité, La mémoire collective à l'épreuve du lignage dans le Jérid tunisien, Paris, La Découverte.

DEPAULE J.C., 1991.- Des espaces qualifiés : présentation, Égypte-Monde arabe, 5 : 7-12.

DePaule J.C. ,1995.- Anthropologie de l'espace, Histoire urbaine, Cahiers PIR villes, Paris, CNRS : 17-73.

Descola Ph., 1986.- La nature domestique, Symbolisme et praxis dans l'écologie des Achuar, Paris, Maison des Sciences de l'Homme. 
Descola PH., 1995.- Les lances du crépuscule, Relations Jivaros, Haute Amazonie, Paris, Plon (Terre Humaine).

DOZY R., 1927.- Supplément aux dictionnaires arabes, Paris, Leide, Maisonneuve Frères, E. J. Brill (2 t.).

Duvignaud J., 1992.- Chebika, Paris, Plon.

GrIAUle M., 1985 [1 $1^{\text {ire } e ́ d . ~ 1948] .-~ D i e u ~ d ' e a u, ~ E n t r e t i e n s ~ a v e c ~ O g o t e m m e ̂ l i, ~ F a y a r d, ~ P a r i s . ~}$

HÉNIA A., 1980.- Le Grîd, ses relations avec le beylik de Tunis (1676-1840), Tunis, Publication de l'Université de Tunis.

KASSAH A., 1996.- Les oasis tunisiennes, Aménagement hydro-agricole et développement en zone aride, Tunis, Publications Cérès, série Géographique, 13, 345 p.

KAZIMIRSKI A. de B., 1860.- Dictionnaire arabe-français, Paris, Maisonneuve et $\mathrm{C}^{\mathrm{ie}}$ éditeur (2 t.).

KILANI M., 1992.- La construction de la mémoire, Le lignage et la sainteté dans l'oasis d'el Ksar, Genève, Labor et Fides.

LeROI-GourhaN A., 1956.- Les domaines de l'esthétique, L'homme, Races et Mœurs, Encyclopédie Clartées, Paris : 1-13.

PATY DE Clam A. (du), 1890.- Fastes chronologiques de Tozeur, Paris, Augustin Challamel.

Puig N., 1997.- Nouvelles sociabilités dans le Sud tunisien, Territoires et formes d'organisation collective à Tozeur, Monde arabe, Maghreb-Machrek, 157 : 78-89.

Rouissi M., 1973.- Une oasis du Sud-tunisien, Le Jarid, Essai d'histoire sociale, Paris, th. EPHE, 350 p. (2 vol.)

\begin{tabular}{|c|c|}
\hline a & 1 \\
\hline $\mathrm{b}$ & - \\
\hline$t$ & $ت$ \\
\hline th & $\therefore$ \\
\hline j & $z$ \\
\hline $\mathrm{h}^{\prime}$ & 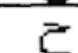 \\
\hline $\mathrm{kh}$ & $\dot{0}$ \\
\hline $\mathrm{d}$ & 2 \\
\hline $\mathrm{d}$. & $\dot{3}$ \\
\hline r & 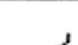 \\
\hline z & 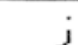 \\
\hline s & سرى \\
\hline sh & لريى \\
\hline $\mathrm{s}^{\prime}$ & \\
\hline
\end{tabular}

\begin{tabular}{|c|c|}
\hline $\mathrm{dh}$ & ض \\
\hline$t^{\prime}$ & 5 \\
\hline$z^{\prime}$ & E \\
\hline “ & $\varepsilon$ \\
\hline gh & $\dot{\varepsilon}$ \\
\hline q ou g & ف \\
\hline $\mathrm{k}$ & $\xi$ \\
\hline 1 & $J$ \\
\hline $\mathrm{m}$ & 5 \\
\hline n & ن ن \\
\hline h & 0 \\
\hline$w$ & $g$ \\
\hline y & s \\
\hline
\end{tabular}

Document 8 : Transcription utilisée des caractères arabes. 
ISSN 0183-5173

Muséum National d'Histoire Naturelle, 57, rue Cuvier 75231 Paris cedex 05

E. BERNUS - Noms composés donnés aux herbes par les Touaregs

V. BATTESTI et N. PUIG - Le sens des lieux. Espaces et pratiques dans les palmeraies du Jérid (Sud-ouest tunisien)

N. EL ALAOUI - Paysages, usages et voyages d'Argania spinosa (L.) Skeels $\left(\mathrm{IX}^{\mathrm{e}}-\mathrm{X}^{\mathrm{e}}\right.$ siècles $)$

Fr. DE LANFRANCHI, BUI THI MAI et M. GIRARD - La fabrication d'huile de lentisque (Linsticu ou chessa) en Sardaigne

É. CHOUVIN - Jardin d'Église en Éthiopie centrale 101

S. CHAFFARD - Rôle des chiens de chasse chez les Konon des Monts Nimba : à l'interface entre le village et la brousse

A. SOMÉ, D.-Y. ALEXANDRE et V. HIEN - Bio-indicateurs paysans de la fertilité des sols et gestion du cycle culture-jachère en zone soudanienne (Burkina Faso)

J. SUEUR - Les insectes Hémiptères représentés sur les urnes dynastiques de Huê (Viêt-nam)

É. MOLLARD - Les hortillonnages en Thaïlande et dans le monde

A. WALTER et F. TZÉRIKIANTZ - La tarodière irriguée : un système d'agriculture diversifiée

C. BENOIT - Les jardins de la Caraibe : lieux d'histoire et de territoire ?

L'exemple de la Guadeloupe

P. SALAÛNN - Le système de production agricole hmong à Saül (Guyane française) : modalités de pérennisation

J.-F. MAUFFREY - Caractérisation d'un prélèvement cynégétique de subsistance sur un chantier de route forestière de Guyane française .... 